\title{
THE EFFECTIVE STRATEGY FOR CALCULATING STRAINS AND STRESSES IN ELASTO-PLASTIC TORSION OF A BAR
}

\author{
Eugeniusz Zieniuk, Agnieszka Bo€tuć \\ University of Bialystok, Faculty of Mathematics and Computer Science, Bialystok, Poland \\ e-mail: ezieniuk@ii.uwb.edu.pl; aboltuc@ii.uwb.edu.pl
}

\begin{abstract}
The paper presents the general approximation strategy for derivatives of solutions obtained by the parametric integral equation system (PIES). The proposed approach is applied to calculating derivatives in linear and nonlinear torsion of a bar. The nonlinear problem is solved iteratively and requires repeated calculating of integrals in order to determine stresses or strains. Due to elimination of the repeated integration, the proposed strategy increases the efficiency of calculations. Furthermore, it ensures high accuracy of solutions to the considered problem, which has been tested in comparison to the analytical and/or numerical solutions reported in the literature obtained by other methods.
\end{abstract}

Keywords: PIES, torsion of a bar, approximation, stresses, strains

\section{Introduction}

The parametric integral equation system (PIES) is a method developed as an alternative to other well-known numerical methods as the finite element method (FEM) (Ameen, 2005; Zienkiewicz, 1977), boundary element method (BEM) (Aliabadi, 2002; Ameen, 2005) or so-called meshless methods (Liu, 2002). This method has confirmed its advantages in solving boundary value problems modelled by various linear partial differential equations: Laplace's (Zieniuk, 2001), Helmholtz's (Zieniuk and Bołtuć, 2006a), Navier-Lamé's (Zieniuk and Bołtuć, 2006b; Zieniuk, 2013; Bołtuć and Zieniuk, 2011, 2013). Successfully verified benefits are as follows: no discretization of the boundary and the domain which is replaced by effective modelling using curves and surfaces, high accuracy of obtained solutions, lower computational complexity. These advantages are very encouraging to generalize the method to other problems, including more complex (e.g. nonlinear).

The initial attempts to generalize and apply the PIES method for solving nonlinear boundary value problems in mechanics showed the authors the type and scale of problems that occur during their application. The first is the necessity of repeated calculating of integrals, often singular, in order to determine the results (often derivatives of the solutions) at many points of the domain. Accurate computation of singular integrals (even with a strong singularity, e.g. in the elastoplastic mechanical problems) is a serious problem, which determines the effectiveness of the whole PIES method (Zieniuk, 2013). It should be mentioned that these problems are not more significant than in BEM. Moreover, the numerically computed solutions and especially their derivatives obtained at points close to the boundary are less accurate because the singularities occur in the vicinity of the boundary. Errors generated in these cases, accumulated in an iterative process used for solving the nonlinear problems, affect the accuracy of the final results.

Therefore, in the research carried out by the authors, the new strategy has been searched, which can eliminate the above mentioned drawbacks. The authors have developed the strategy based on the interpolation of solutions obtained directly from PIES and the integral identity, and differentiation of the so-obtained interpolation polynomial. On the basis of the obtained 
interpolation polynomial, we can determine derivatives of any order with respect to any variable in a simple manner using a single set of values (solutions on the boundary and in the domain). The thus obtained derivatives of the polynomial allow calculating derivatives in a continuous way at any point of the domain, and also the boundary. Verification of the mentioned strategy on the linear elasticity problems is presented by Bołtuć and Zieniuk (2013). It should be emphasized that the proposed strategy can be applied also in BEM, but it does not guarantee the highest efficiency. About the effectiveness of the strategy firstly decides the way of modelling the considered domain and the resulting number of algebraic equations to be solved in order to achieve the interpolation. On the other hand, on the accuracy of the approximation the greatest impact has the optimal arrangement of interpolation nodes at which the solutions are obtained. The effectiveness of performing the interpolation determines freedom in automatic generation of an optimal nodes arrangement. Such an arrangement in combination with PIES is shown in the following paper.

Due to the complexity of nonlinear problems, we decided to verify PIES with the approximation strategy on the most elementary problem in the field of nonlinear solid mechanics. Therefore, the verification of the presented strategy has been done on nonlinear torsion of the bar. The elementariness of this issue as compared to other nonlinear problems ensures proper verification of the mathematical apparatus applied to the proposed strategy. Numerical solution of the elasto-plastic bar torsion has been frequently studied (including inverse problems) using methods such as the finite difference method (FDM) (Mendelson, 1968), FEM (Mamedov, 1995; Yamada et al., 1972), BEM (Mendelson, 1975; Sapountzakis and Tsipiras, 2009) and recently using the method of fundamental solutions (MFS) (Kołodziej and Gorzelańczyk, 2012), which significantly facilitates the verification of the results obtained by the applied strategy.

The purpose of this paper is to apply the strategy for approximation of derivatives of solutions and to verify its effectiveness on the example of the elasto-plastic torsion problem solved by PIES. This means that on the basis of the numerical solutions to the elasto-plastic torsion problem obtained by the PIES method and using the proposed approximation strategy, we calculate strains and stresses. The analytical solution in the considered example exists only for the elastic torsion. In the case of plasticity, we are dealing only with numerical solutions, but this elementary problem has been solved by various methods, which results in better verification. First, the problem has been solved as linear, allowing one to verify the PIES method with analytical solutions. Then, the effectiveness of the strategy has been tested in an iterative process used to solve the nonlinear problems, where obtained results have been compared with other numerical solutions.

\section{Formulation of problem}

Consider a prismatic bar subjected to a twisting moment as shown in Fig. 1.

The elasto-plastic problem of torsion can be formulated in several ways. In this paper, we use a formulation in terms of the warping function, because it is physically most meaningful and most universal, and we consider bars of rectangular cross-sections. The main equations are as follows (Mendelson, 1975):

a) The equilibrium equation

Assume in accordance with the bar torsion theory that the stress state is defined independently of $x_{3}$ by components $\tau_{13}$ and $\tau_{23}$ (Fig. 1b), and the remaining elements are zero $\sigma_{11}=\sigma_{22}=\sigma_{33}=\tau_{12}=0$. The equilibrium differential equations in this case are reduced to one (Sokolowski, 1957)

$$
\frac{\partial \tau_{13}}{\partial x_{1}}+\frac{\partial \tau_{23}}{\partial x_{2}}=0
$$

where $\tau_{13}$ and $\tau_{23}$ are the shear stresses acting on the cross-section. 


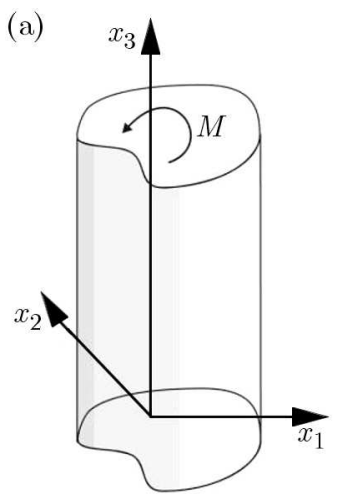

(b)

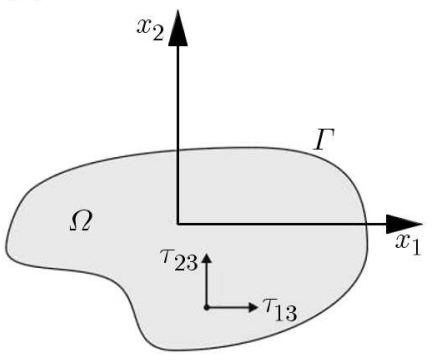

Fig. 1. (a) Prismatic bar under the action of a twisting moment, and (b) the cross-section with the shear stresses

b) Stress-strain relations

$$
\tau_{13}=2 G\left(\varepsilon_{13}-\varepsilon_{13}^{p}\right) \quad \tau_{23}=2 G\left(\varepsilon_{23}-\varepsilon_{23}^{p}\right)
$$

where $G$ is the shear modulus, $\varepsilon_{13}$ and $\varepsilon_{23}$ are the total strains and $\varepsilon_{13}^{p}$ and $\varepsilon_{23}^{p}$ are the plastic shear strains.

c) Saint-Venant's relations

$$
\varepsilon_{13}=\frac{1}{2}\left(-\alpha x_{2}+\frac{\partial w}{\partial x_{1}}\right) \quad \varepsilon_{23}=\frac{1}{2}\left(\alpha x_{1}+\frac{\partial w}{\partial x_{2}}\right)
$$

where $\alpha$ is the angle of twist per unit length and $w$ is the warping function. The warping function is assumed to be the same for all cross-sections, therefore it is a function of $x_{1}$ and $x_{2}$ only (Ameen, 2005).

Substituting equations (2.3) into (2.2) and the result into (2.1), we obtain

$$
\frac{\partial^{2} w}{\partial x_{1}^{2}}+\frac{\partial^{2} w}{\partial x_{2}^{2}}=F\left(x_{1}, x_{2}\right) \quad \text { in } \quad \Omega
$$

where $\Omega$ is the region of the cross-section and

$$
F\left(x_{1}, x_{2}\right)=2\left(\frac{\partial \varepsilon_{13}^{p}}{\partial x_{1}}+\frac{\partial \varepsilon_{23}^{p}}{\partial x_{2}}\right)
$$

The plastic strains appearing in (2.2) and (2.5) can be presented by the following expressions

$$
\varepsilon_{13}^{p}=\frac{\varepsilon_{p}}{\varepsilon_{e t}} \varepsilon_{13} \quad \varepsilon_{23}^{p}=\frac{\varepsilon_{p}}{\varepsilon_{e t}} \varepsilon_{23}
$$

where

$$
\varepsilon_{e t}=\frac{2}{\sqrt{3}} \sqrt{\varepsilon_{13}^{2}+\varepsilon_{23}^{2}} \quad \varepsilon_{p}=\frac{\varepsilon_{e t}-\frac{2}{3}(1+\nu) \varepsilon_{0}}{1+\frac{2}{3}(1+\nu) \frac{m}{1-m}}
$$

in the case of linear strain hardening, where $\varepsilon_{0}$ is the yield strain, $m$ is the linear strain hardening parameter and $\nu$ Poisson's ratio. Formula $(2.7)_{2}$ holds for $0 \leqslant m<1$, where $m$ is equal to 0 for a perfectly plastic case. In an elastic case, $m$ is equal to 1 and $\varepsilon_{p}=0$.

Calculation of the function defined by formula (2.5) requires the derivatives of the plastic strains. Therefore, we should differentiate expression $(2.6)_{1}$ with respect to the $x_{1}$-variable, obtaining the equation

$$
\frac{\partial \varepsilon_{13}^{p}}{\partial x_{1}}=\frac{B \varepsilon_{13} \frac{\partial \varepsilon_{e t}}{\partial x_{1}}}{A \varepsilon_{e t}^{2}}+\left(\frac{\varepsilon_{e t}-B}{A \varepsilon_{e t}}\right) \frac{\partial \varepsilon_{13}}{\partial x_{1}}
$$


where $\varepsilon_{e t}$ and $\varepsilon_{13}$ are expressed by formulas $(2.7)_{1}$ and $(2.3)_{1}$, and the remaining functions are defined using the following expressions

$$
\begin{array}{ll}
\frac{\partial \varepsilon_{e t}}{\partial x_{1}}=\frac{4}{3 \varepsilon_{e t}}\left(\varepsilon_{13} \frac{\partial \varepsilon_{13}}{\partial x_{1}}+\varepsilon_{23} \frac{\partial \varepsilon_{23}}{\partial x_{1}}\right) & \frac{\partial \varepsilon_{13}}{\partial x_{1}}=\frac{1}{2} \frac{\partial^{2} w}{\partial x_{1}^{2}} \\
\frac{\partial \varepsilon_{23}}{\partial x_{1}}=\frac{1}{2}\left(\alpha+\frac{\partial^{2} w}{\partial x_{1} \partial x_{2}}\right) &
\end{array}
$$

and

$$
A=1+\frac{2}{3}(1+\nu) \frac{m}{1-m} \quad B=\frac{2}{3}(1+\nu) \varepsilon_{0}
$$

In the same manner, we obtain the derivative of $\varepsilon_{23}^{p}(2.6)_{2}$ with respect to $x_{2}$.

The boundary conditions for the described above approach and the considered in the paper rectangular boundary $\Gamma$ parallel to one of the coordinate axes can be presented in the following form

$$
\frac{\partial w}{\partial n}=\alpha\left(n_{1} x_{2}-n_{2} x_{1}\right) \quad \text { on } \quad \Gamma
$$

where $n_{1}$ and $n_{2}$ are the direction cosines of the external normal to the boundary with respect to the $x_{1}$ and $x_{2}$ axes, respectively.

\section{PIES for the elasto-plastic bar torsion problem}

\subsection{Modeling of the boundary and the domain}

Developed by the authors PIES is an analytical modification of the boundary integral equation (BIE). This modification involves direct inclusion of the boundary shape defined in a general way in the formalism of the equation. Such an approach allows separation of the approximation of the boundary shape from the approximation of boundary functions, and thus makes it possible to independently increase the accuracy and efficiency of these two elements. From the very beginning, the main advantage of the PIES method is the elimination of the classical discretization by elements understood in the manner known from FEM or BEM methods. To create the shape of the boundary we use any parametric curves, while in the case of modeling also the domain (as in the present problem) surface patches are used. Figure $2 \mathrm{~b}$ presents an
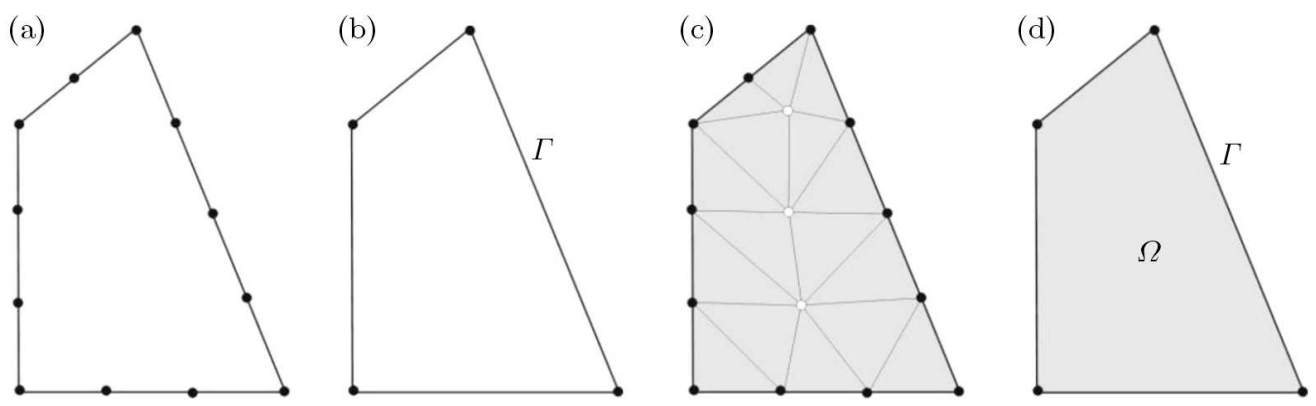

Fig. 2. Modelling of the boundary: (a) in BEM by boundary elements using nodes $\bullet$, (b) in PIES by four Bézier curves using control points $\bullet$, and the domain: (c) in BEM by cells using nodes $\bullet$ and $\circ$, (d) in PIES by Bézier bilinear surface using control points

exemplary way of boundary modeling by curves of the first degree, while the modeling of the domain using the bilinear surface is presented in Fig. 2d. Considering curvilinear shapes, we have to use curves and patches of higher degrees. Both curves and patches are well-known and 
efficient computer graphics tools (Foley et al., 1994), the advantages of which are used in PIES. It should be noted, however, that these curves or patches are not used in PIES as a different kind of elements known from the element methods. The modeling of the shape in PIES should be treated as global, because the number of used curves or patches depends only on the complexity of the shape. Considering, for example, any quadrangle, the definition of the domain requires only one surface. The type of the surface depends on the type of the boundary that surrounds the domain. In the case of the quadrangle, we use the bilinear surface, while the area with the curvilinear boundary is defined efficiently using the bicubic patch. Dealing with more complex shapes (e.g. polygons) we should join the surfaces. An example of the modeling of the boundary and the domain in PIES is presented in Fig. 2b,d. For comparison Fig. 2a,c presents the way of modeling in the BEM method.

\subsection{Fundamentals of the PIES method}

As mentioned earlier, PIES for various differential equations have been obtained by the analytical modification of BIE, like it is shown in (Zieniuk, 2001, 2013; Zieniuk and Bołtuć, 2006a,b). PIES applied to solve the elasto-plastic torsion of a bar (as multiple solution of the Poisson equation) is obtained in the same way, and for a smooth boundary can be presented as

$$
\frac{1}{2} u_{l}\left(s_{1}\right)=\sum_{j=1}^{n} \int_{s_{j-1}}^{s_{j}}\left[\bar{U}_{l j}^{*}\left(s_{1}, s\right) p_{j}(s)-\bar{P}_{l j}^{*}\left(s_{1}, s\right)_{j}(s)\right] J_{j}(s) d s+\int_{\Omega} \overline{\bar{U}}_{l}^{*}\left(s_{1}, \mathbf{y}\right) F(\mathbf{y}) d \Omega(\mathbf{y})
$$

where $\mathbf{y}=\left[y_{1}, y_{2}\right] \in \Omega, J_{j}(s)$ is the Jacobian of the transformation

$$
J_{j}(s)=\sqrt{\left(\frac{\partial \Gamma_{j}^{(1)}(s)}{\partial s}\right)^{2}+\left(\frac{\partial \Gamma_{j}^{(2)}(s)}{\partial s}\right)^{2}}
$$

$l=1,2, \ldots, n$ and $n$ is the number of segments that make the boundary. The parametric variables $s, s_{1}$ satisfy the following relations $\bar{s}_{l-1} \leqslant s_{1} \leqslant \bar{s}_{l}, \bar{s}_{j-1} \leqslant s \leqslant \bar{s}_{j}$, where $\bar{s}_{l-1}$ and $\bar{s}_{j-1}$ are the beginnings of the segments $l, j$, respectively, while $\bar{s}_{l}, \bar{s}_{j}$ are their ends.

Using equation (3.1) we can solve equation (2.4) and obtain solutions on the boundary. Therefore, $u_{l}\left(s_{1}\right)(l=1,2, \ldots, n)$ in (3.1) corresponds to the function $w\left(x_{1}, x_{2}\right)$ in (2.4) for $x_{1}, x_{2} \in \Gamma$.

The first and second integrands in (3.1) are the boundary fundamental solution and boundary singular solution to the Laplace equation. They can be presented as (Zieniuk, 2001)

$$
\bar{U}_{l j}^{*}\left(s_{1}, s\right)=\frac{1}{2 \pi} \ln \frac{1}{\sqrt{\eta_{1}^{2}+\eta_{2}^{2}}} \quad \bar{P}_{l j}^{*}\left(s_{1}, s\right)=\frac{1}{2 \pi} \frac{\eta_{1} n_{1}(s)+\eta_{2} n_{2}(s)}{\eta_{1}^{2}+\eta_{2}^{2}}
$$

where $\eta_{1}=\Gamma_{l}^{(1)}\left(s_{1}\right)-\Gamma_{j}^{(1)}(s)$ and $\eta_{2}=\Gamma_{l}^{(2)}\left(s_{1}\right)-\Gamma_{j}^{(2)}(s)$, while $n_{1}(s)$ and $n_{2}(s)$ are the direction cosines of the external normal to the boundary. The shape of the boundary defined by any parametric curves $\boldsymbol{\Gamma}(s)=\left[\Gamma^{(1)}(s), \Gamma^{(2)}(s)\right]^{\mathrm{T}}$ is included into mathematical formalism of formulas (3.2). The types of curves and formulas describing them can be found by Foley et al. (1994).

The integrand in the integral over the domain is presented by the following expression, also known as the Poisson equation

$$
\overline{\bar{U}}_{l}^{*}\left(s_{1}, \mathbf{y}\right)=\frac{1}{2 \pi} \ln \frac{1}{\sqrt{\bar{\eta}_{1}^{2}+\bar{\eta}_{2}^{2}}}
$$

where $\bar{\eta}_{1}=\Gamma_{l}^{(1)}\left(s_{1}\right)-y_{1}, \bar{\eta}_{2}=\Gamma_{l}^{(2)}\left(s_{1}\right)-y_{2}$. 
In order to obtain the solutions $u$ in the domain $\Omega$ (at point $\mathbf{x}=\left[x_{1}, x_{2}\right]$ ) we use the integral identity

$$
u(\mathbf{x})=\sum_{j=1}^{n} \int_{s_{j-1}}^{s_{j}}\left[\hat{\bar{U}}_{j}^{*}(\mathbf{x}, s) p_{j}(s)-\hat{\bar{P}}_{j}^{*}(\mathbf{x}, s) u_{j}(s)\right] J_{j}(s) d s+\int_{\Omega} \hat{\overline{\bar{U}}}^{*}(\mathbf{x}, \mathbf{y}) F(\mathbf{y}) d \Omega(\mathbf{y})
$$

where the integrands are presented by

$$
\begin{array}{ll}
\hat{\bar{U}}_{j}^{*}(\mathbf{x}, s)=\frac{1}{2 \pi} \ln \frac{1}{\sqrt{\ddot{r}_{1}^{2}+\ddot{r}_{2}^{2}}} \quad \hat{\bar{P}}_{j}^{*}(\mathbf{x}, s)=\frac{1}{2 \pi} \frac{\ddot{r}_{1} n_{1}(s)+\ddot{r}_{2} n_{2}(s)}{\ddot{r}_{1}^{2}+\ddot{r}_{2}^{2}} \\
\hat{\bar{U}}^{*}(\mathbf{x}, \mathbf{y})=\frac{1}{2 \pi} \ln \frac{1}{\sqrt{\hat{\tilde{r}}_{1}^{2}+\hat{\ddot{r}}_{2}^{2}}} &
\end{array}
$$

and $\ddot{r}_{1}=x_{1}-\Gamma_{j}^{(1)}(s), \ddot{r}_{2}=x_{2}-\Gamma_{j}^{(2)}(s), \hat{\vec{r}}_{1}=x_{1}-y_{1}, \hat{\vec{r}}_{2}=x_{2}-y_{2}, \mathbf{x}, \mathbf{y} \in \Omega$ and $\mathbf{x} \neq \mathbf{y}$. The form of the Jacobian $J_{j}(s)$ is the same as in formula (3.1).

Using equation (3.4) we can obtain the solution to equation (2.4) in the domain. Therefore, $u(\mathbf{x})$ in (3.4) corresponds to the function $w\left(x_{1}, x_{2}\right)$ for $x_{1}, x_{2} \in \Omega$.

\subsection{Numerical solution of PIES}

The solution of PIES represented by formula (3.1) consists of finding unknown boundary functions $u_{j}(s)$ or $p_{j}(s)$ on particular segments of the boundary. These functions are approximated by the approximating series

$$
u_{j}(s)=\sum_{k=0}^{N-1} u_{j}^{(k)} T_{j}^{(k)}(s) \quad p_{j}(s)=\sum_{k=0}^{N-1} p_{j}^{(k)} T_{j}^{(k)}(s) \quad j=1, \ldots, n
$$

where $u_{j}^{(k)}, p_{j}^{(k)}$ are unknown coefficients, $T_{j}^{(k)}(s)$ are the Chebyshev polynomials of the first kind, $N$ is the number of terms in the series on the segment $j$ and $n$ is the number of segments. After imposing the boundary conditions, we can obtain the unknown boundary functions for the individual segments using the approximating form of PIES. This form is obtained after substituting series (3.6) to analytical form of PIES (3.1)

$$
\begin{aligned}
& \frac{1}{2} \sum_{k=0}^{N-1} u_{l}^{(k)} T_{l}^{(k)}\left(s_{1}\right)=\sum_{j=1}^{n} \sum_{k=0}^{N-1}\left[p_{j}^{(k)} \int_{\bar{s}_{j-1}}^{\bar{s}_{j}} \bar{U}_{l j}^{*}\left(s_{1}, s\right)-u_{j}^{(k)} \int_{\bar{s}_{j-1}}^{\bar{s}_{j}} \bar{P}_{l j}^{*}\left(s_{1}, s\right)\right] J_{j}(s) T_{j}^{(k)}(s) d s \\
& \quad+\int_{\Omega} \overline{\bar{U}}_{l}^{*}\left(s_{1}, \mathbf{y}\right) F(\mathbf{y}) d \Omega(\mathbf{y})
\end{aligned}
$$

Writing down equation (3.7) at the collocation points $s_{1}^{i}(i=1,2, \ldots, I), I=n \times N$, we obtain the system of algebraic equations

$$
\mathbf{H u}=\mathbf{G p}+\mathbf{D}
$$

where $\mathbf{H}=[H]_{I \times I}, \mathbf{G}=[G]_{I \times I}$ are the square matrices of elements expressed by integrals over boundary from (3.7), $\mathbf{u}$ and $\mathbf{p}$ are vectors which contain the coefficients of approximation series (3.6), while $\mathbf{D}$ is the vector of elements expressed by the integral over the domain from equation (3.7).

After imposing the boundary conditions and some transformations, equation (3.8) takes the following form

$$
\mathbf{A X}=\mathbf{B}+\mathbf{D}
$$


where the vector $\mathbf{X}$ contains unknown coefficients from (3.7), while the vector $\mathbf{B}$ is known and depends on the given boundary conditions.

The only problem is the vector $\mathbf{D}$ whose components require calculation of the function $F(\mathbf{y})$ in order to make the integration over the domain $\Omega$. The value of this function is unknown and depends on the plastic strains which are a part of the solution. For that reason it is necessary to apply an iterative process and at its first step to solve equation (3.9) with an arbitrary distribution of the function $F(\mathbf{y})$. We have assumed that the function at the first step of the iterative process (according to Fig. 3) takes a constant value (zero) in the entire domain.

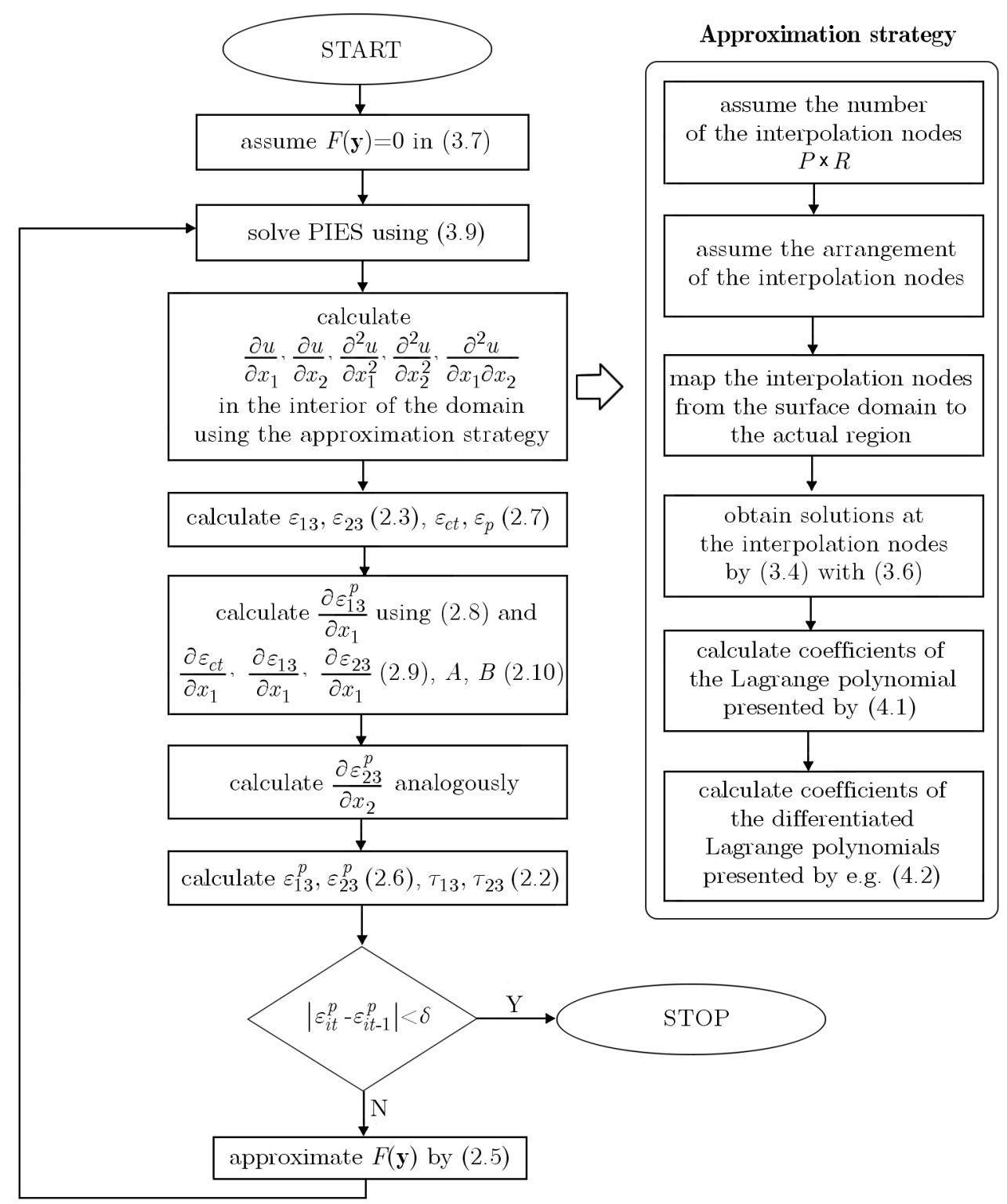

Fig. 3. The diagram of solving the elasto-plastic torsion of the bar

Finally, the solution to equation (3.9) also requires calculation of the integral over the domain which contains $F(\mathbf{y})$. The mentioned at the beginning of this Section way of the modelling gives the possibility for integrating globally over the entire domain without dividing it into cells as it is done in BEM (Aliabadi, 2002; Ameen, 2005). In BEM, the domain is divided into sub-domains of elementary shapes over which integrals are calculated with a small number of weights in the quadrature of numerical integration. Then the values of the so determined integrals are summed. In PIES, the integrals are calculated globally over the entire domain using the Gauss-Legendre 
quadrature with a large number of weights. The results of the global integration in PIES on the example of elasticity problems with body forces can be found among others by Bołtuć and Zieniuk (2011).

The conceptual diagram of the process of solving the elasto-plastic torsion problem is presented in Fig. 3.

After solving (3.9) with the adopted initial value of $F(\mathbf{y})$ we have to compute the warping function at the points of the domain. This is done using integral identity (3.4) after substituting series (3.6) with known or calculated boundary functions. Then the total strains defined by formulas (2.3) are calculated. However, it is clear from formulas (2.3) that the calculations do not require the value of the function $u$ at selected points of the area, but rather the value of its first and second derivative with respect to $x_{1}$ and $x_{2}$. These derivatives can be determined by analytical differentiation of integral identity (3.4). However, such an approach will result in multiple calculations of integrals from identity (3.4) and its derivatives, which is particularly troublesome in an iterative process. Therefore, it has been decided to obtain these derivatives in a more efficient way, using the proposed and described in the next Section approximation strategy. This strategy is also included in the diagram presented in Fig. 3.

Having calculated total strains (2.3) we should determine the plastic strains using formulas (2.6). Then, in order to obtain a better approximation of $F(\mathbf{y})$, we determine its value again using expression (2.5). Again, we solve equation (3.9) and the solution becomes approximated in successive iteration steps until fulfilling the given stop criterion. The iterative process should be recognized as finished if the difference between two lastly obtained values at all considered points is smaller than the convergence criterion $\delta$.

\section{The approximation strategy for derivatives}

The proposed in the paper approximation algorithm initially has been based on the already known from PIES series used in the boundary function approximation, and in fact has been their generalization to a series dependent on two variables. They are easily differentiable, hence there occurs the possibility of direct calculation of the solution derivatives of any order with respect to any variable. It should be emphasized that the derivatives are obtained in a continuous way at any point of the area and the boundary. Tests of the approach based on the approximation series considering the example of linear elasticity problems are presented by Bołtuć and Zieniuk (2013).

Carrying out the research, we noticed a few shortcomings. The most important is illconditioning of the system of algebraic equations solved during approximation. The explicit form of this system is presented in the paper (Bołtuć and Zieniuk, 2013). Therefore, various improvements have been introduced, which are described in detail in (Bołtuć and Zieniuk, 2013). The most important is application of the 2D Lagrange polynomial to the approximation of the derivative of the solutions. Using it, we can eliminate the necessity of solving the ill-conditioned system of equations. This is particularly important in problems solved iteratively, because illconditioning does not guarantee the stability of solutions for even small disorders of the vector with constant terms (the right-hand side vector).

In this paper, we focus only on general describing the proposed technique. Details and results of the research on its efficiency and accuracy can be found in (Bołtuć and Zieniuk, 2013). The steps of the algorithm are as follows (they are also presented in Fig. 3):

K1 - Assume the number $(P \times R)$ of the interpolation nodes.

K2 - Assume the distribution of interpolation nodes; different node distributions in the square $(P=R=9)$ are presented in Fig. 4 and their impact on the quality of the obtained solutions is described in details in (Bołtuć and Zieniuk, 2013). Figure 4 also shows the 

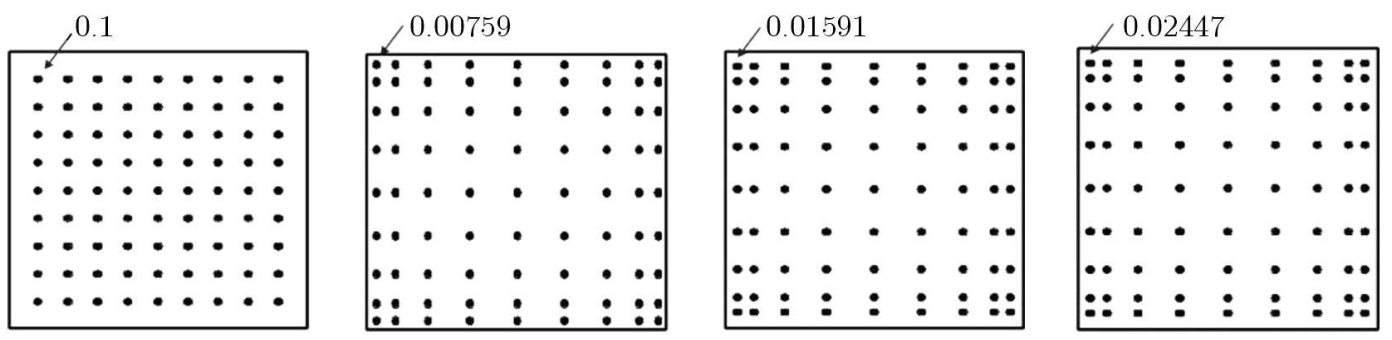

Fig. 4. Variants of the arrangement of interpolation nodes: (a) uniform, (b) at roots of the Chebyshev polynomial of the first kind, (c) at roots of the Legendre polynomial, (d) at roots of the Chebyshev polynomial of the second kind

distance of the extreme interpolation node from the boundary of the unit square for various node distributions.

K3 - Map the interpolation nodes from the area in which they are arranged (the unit square) to the actual area using mathematical expressions describing surfaces (Foley et al., 1994). Such a situation is possible because of the fact that the mentioned unit area is also the domain of polynomials describing the surfaces. An example of mapping the interpolation nodes from the unit square to the actual shape of the considered area is shown in Fig. 5. This is an original, efficient and automatic way of optimal arrangement of the interpolation nodes with the help of surface patches.
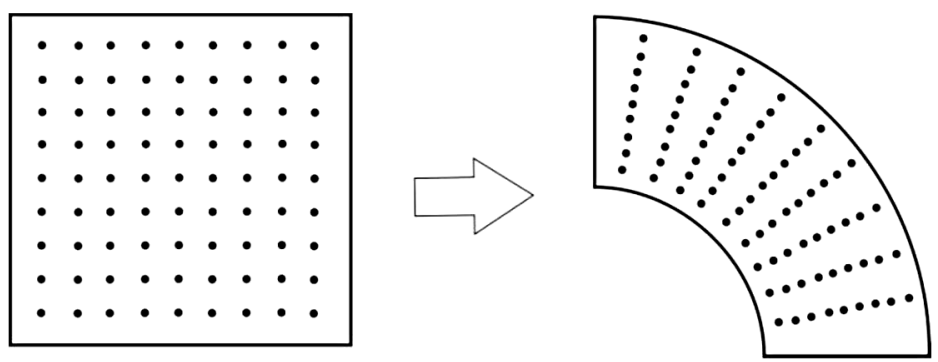

Fig. 5. Mapping the interpolation nodes from the domain of the surface to the actual area

$\mathbf{K} 4$ - Obtain solutions $u(\mathbf{x})$ at the interpolation nodes using the integral identity (3.4).

K5 - Create the 2D Lagrange polynomial, which is a generalization of the 1D case and has the following form

$$
W_{u}\left(x_{1}, x_{2}\right)=\sum_{p=0}^{P-1} \sum_{r=0}^{R-1} u\left(x_{1 p}, x_{2 r}\right) L_{p r}\left(x_{1}, x_{2}\right)
$$

where

$$
\begin{aligned}
& L_{p r}\left(x_{1}, x_{2}\right)=L_{p}\left(x_{1}\right) L_{r}\left(x_{2}\right) \\
& L_{p}\left(x_{1}\right)=\prod_{g=0, g \neq p}^{P-1} \frac{x_{1}-x_{1 g}}{x_{1 p}-x_{1 g}} \quad L_{r}\left(x_{2}\right)=\prod_{g=0, g \neq r}^{R-1} \frac{x_{2}-x_{2 g}}{x_{2 r}-x_{2 g}}
\end{aligned}
$$

and $u\left(x_{1 p}, x_{2 r}\right)$ are values of the warping function at $P \times R$ given (declared in step K2) points of the area and the boundary; the polynomial $W_{u}\left(x_{1}, x_{2}\right)$ approximates function $w$ from (2.3) and (2.4). 
K6 - Direct differentiation of the resulting polynomial with respect to each variable in order to obtain required partial derivatives. As a result, we obtained polynomials which approximate the derivatives of solutions, e.g.

$$
\begin{aligned}
& \frac{\partial W_{u}\left(x_{1}, x_{2}\right)}{\partial x_{1}}=\sum_{p=0}^{P-1} \sum_{r=0}^{R-1} u\left(x_{1 p}, x_{2 r}\right) \frac{\partial L_{p r}\left(x_{1}, x_{2}\right)}{\partial x_{1}} \\
& \frac{\partial L_{p r}\left(x_{1}, x_{2}\right)}{\partial x_{1}}=\frac{\partial L_{p}\left(x_{1}\right)}{\partial x_{1}} L_{r}\left(x_{2}\right) \\
& \frac{\partial L_{p}\left(x_{1}\right)}{\partial x_{1}}=\sum_{h=1, h \neq p}^{P-1} \frac{1}{x_{1 p}-x_{1 h}} \prod_{z=1, z \neq p, z \neq h}^{P-1} \frac{x_{1}-x_{1 z}}{x_{1 p}-x_{1 z}} \\
& \frac{\partial^{2} W_{u}\left(x_{1}, x_{2}\right)}{\partial x_{1}^{2}}=\sum_{p=0}^{P-1} \sum_{r=0}^{R-1} u\left(x_{1 p}, x_{2 r}\right) \frac{\partial^{2} L_{p r}\left(x_{1}, x_{2}\right)}{\partial x_{1}^{2}} \\
& \frac{\partial^{2} L_{p r}\left(x_{1}, x_{2}\right)}{\partial x_{1}^{2}}=\frac{\partial^{2} L_{p}\left(x_{1}\right)}{\partial x_{1}^{2}} L_{r}\left(x_{2}\right) \\
& \frac{\partial^{2} L_{p}\left(x_{1}\right)}{\partial x_{1}^{2}}=\sum_{h=1, h \neq p}^{P-1} \frac{1}{x_{1 p}-x_{1 h}} \sum_{\substack{o=1, o \neq h, o \neq p}}^{P-1} \frac{1}{x_{1 p}-x_{1 o}} \prod_{\substack{z=1, z \neq p, z \neq h, z \neq 0}}^{P-1} \frac{x_{1}-x_{1 z}}{x_{1 p}-x_{1 z}}
\end{aligned}
$$

The derivatives with respect to the remaining variables are obtained in an analogous way. As shown in Fig. 3, the differentiated polynomials are used further to determine the warping function derivatives, and these derivatives to calculate the derivatives of plastic strains and finally the function $F(\mathbf{y})$ as a result.

\section{Analysis of results}

We consider the problem of the elasto-plastic torsion of a bar of square cross-section with side length $2 a$. Due to double symmetry, only a quarter of the domain has been taken into account (Fig. 6). The shape of the considered square domain is defined using one bilinear Bézier surface. This requires only four corner points $P_{0}, P_{1}, P_{2}, P_{3}$ of the surface.

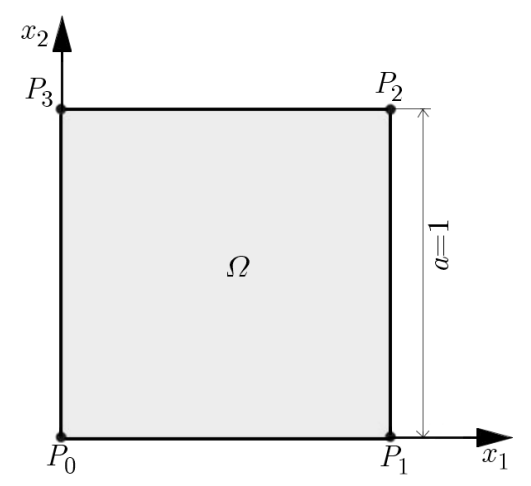

Fig. 6. The quarter of cross-section of the bar

In calculations, the following values have been assumed: $a=1, \nu=0.3$, whilst the dimensionless angle of twist per unit length $\beta=\alpha a / \varepsilon_{0}$ is increased in steps of one from $\beta=1$ to $\beta=6$. The strain hardening parameter $m$ is taken as 0 (perfect plasticity), 0.1, 0.2 and 1 (elasticity).

Initially, we considered torsion of an elastic bar, and therefore $\beta=1$ and $m=1$ have been adopted. This problem has an analytical solution for $\tau_{1}=\tau_{13} /\left(2 G \varepsilon_{0}\right)$ (Sokolnikoff, 1956), 
which can be calculated also numerically using (2.2). Equations (2.3) contain the first derivative of $w$ with respect to $x_{1}$, so to determine the shear stresses we could use the proposed strategy for approximation of the derivative. In order to use it, we have chosen 64 interpolation nodes $(P=R=8)$ distributed at places corresponding to the roots of 8th Chebyshev polynomial of the second kind (Fig. 7).

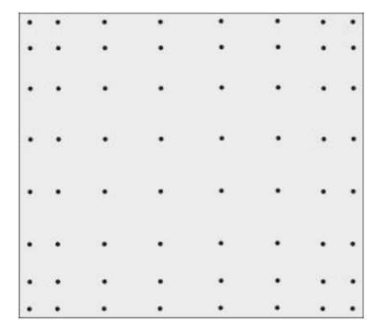

Fig. 7. The arrangement of the interpolation nodes $(P=R=8)$

Taking into account the values of $w$ (obtained by 3.4) at these points as well as their coordinates, we can determine Lagrange polynomial (4.1) and, analogously to (4.2), differentiate it. Then these derivatives are used to determine the stresses presented by $(2.2)_{1}$. The obtained in the described way results at selected points of the domain and compared to analytical solutions are presented in Table 1.

Table 1. Comparison of the exact elastic $\tau_{1}$ with the obtained by the proposed strategy

\begin{tabular}{|c|c|c|c|}
\hline$\left(x_{1}, x_{2}\right)$ & Exact & PIES & $\begin{array}{c}\text { Relative } \\
\text { error [\%] }\end{array}$ \\
\hline \hline$(0.2,0.2)$ & 0.0971 & 0.097081 & 0.01957 \\
\hline$(0.4,0.2)$ & 0.0839 & 0.083950 & 0.05959 \\
\hline$(0.6,0.2)$ & 0.0623 & 0.062312 & 0.01926 \\
\hline$(0.8,0.2)$ & 0.0333 & 0.033380 & 0.24024 \\
\hline$(0.2,0.4)$ & 0.2030 & 0.202945 & 0.02709 \\
\hline$(0.4,0.4)$ & 0.1760 & 0.176557 & 0.31647 \\
\hline$(0.6,0.4)$ & 0.1320 & 0.132236 & 0.17878 \\
\hline$(0.8,0.4)$ & 0.0714 & 0.071442 & 0.05882 \\
\hline$(0.6,0.6)$ & 0.2190 & 0.219405 & 0.18493 \\
\hline$(0.6,0.8)$ & 0.3380 & 0.338050 & 0.01479 \\
\hline$(0.8,0.6)$ & 0.1210 & 0.121260 & 0.21487 \\
\hline$(0.8,0.8)$ & 0.1980 & 0.197739 & 0.13181 \\
\hline
\end{tabular}

As shown in Table 1, the solutions obtained by means of PIES and the proposed algorithm of approximation are characterized by a very high accuracy. A very important advantage is also the fact that if we want to obtain the value of the derivative of another order or with respect to another variable, we can use the same Lagrange polynomial, just differentiate it according to current needs. This avoids repeated calculation of the integrals from analytically differentiated integral identity (3.4).

Another very interesting and useful advantage of the strategy is the ability to determine the derivatives of solutions on the boundary, again by the previously obtained Lagrange polynomial. In the case of the classical approach, obtaining stresses on the boundary is possible using the derivative of the integral identity, wherein there is a singularity arising from the fact that $\mathbf{x} \rightarrow \Gamma$. Using the proposed technique, derivatives of any order with respect to any variable at any point of the boundary and area are calculated using the same expression. The stress values at selected 
points along the boundary compared with the exact solutions (Sokolnikoff, 1956) are presented in Table 2 .

Table 2. Values of $\tau_{1}$ obtained on the boundary

\begin{tabular}{|c|c|c|c|}
\hline$\left(x_{1}, x_{2}\right)$ & Exact & PIES & $\begin{array}{c}\text { Relative } \\
\text { error [\%] }\end{array}$ \\
\hline \hline$(0.0,0.2)$ & 0.101 & 0.101450 & 0.44554 \\
\hline$(0.0,0.4)$ & 0.212 & 0.211629 & 0.17500 \\
\hline$(0.0,0.6)$ & 0.339 & 0.339247 & 0.07286 \\
\hline$(0.0,0.8)$ & 0.492 & 0.492385 & 0.07825 \\
\hline$(0.0,1.0)$ & 0.675 & 0.674900 & 0.01481 \\
\hline$(0.2,1.0)$ & 0.658 & 0.658401 & 0.06094 \\
\hline$(0.4,1.0)$ & 0.605 & 0.605372 & 0.06148 \\
\hline$(0.6,1.0)$ & 0.507 & 0.506964 & 0.00710 \\
\hline$(0.8,1.0)$ & 0.342 & 0.342750 & 0.21929 \\
\hline
\end{tabular}

As can be seen in Table 2, solutions obtained by the approximation strategy are very similar to the analytical results, relative errors do not exceed $0.5 \%$. In order to confirm the reliability and accuracy of the approach, we also examined the dimensionless moment $M^{*}=M /\left(2 G \varepsilon_{0} a^{3}\right)$, where $M=\iint_{\Omega}\left(x_{1} \tau_{23}-x_{2} \tau_{13}\right) d x_{1} d x_{2}$ and the dimensionless maximum shear stress $\tau_{\text {max }}^{*}=$ $\tau_{\max } /\left(2 G \varepsilon_{0}\right)$, where $\tau_{\max }=\left(\sqrt{\tau_{13}^{2}+\tau_{23}^{2}}\right)_{\max }$. The obtained by PIES results have been compared with the exact solutions and are presented in Table 3.

Table 3. Comparison of $M^{*}$ and $\tau_{\max }^{*}$ obtained by PIES with the exact results

\begin{tabular}{|c|c|c|c|}
\hline & Exact & PIES & $\begin{array}{c}\text { Relative } \\
\text { error [\%] }\end{array}$ \\
\hline \hline$M^{*}$ & 1.125 & 1.12463 & 0.0328 \\
\hline$\tau_{\max }^{*}$ & 0.6754 & 0.67490 & 0.0740 \\
\hline
\end{tabular}

Table 3 presents the high accuracy of the proposed approach.

The last test carried out for the elastic case concerned the comparison of the dimensionless moment $M^{*}$ for $m=1$ and different values of $\beta$. These results, together with the analytical solutions and obtained by the methods: boundary integral method (BIM) (Mendelson, 1975) and MFS (Kołodziej and Gorzelańczyk, 2012) are presented in Fig. 8.

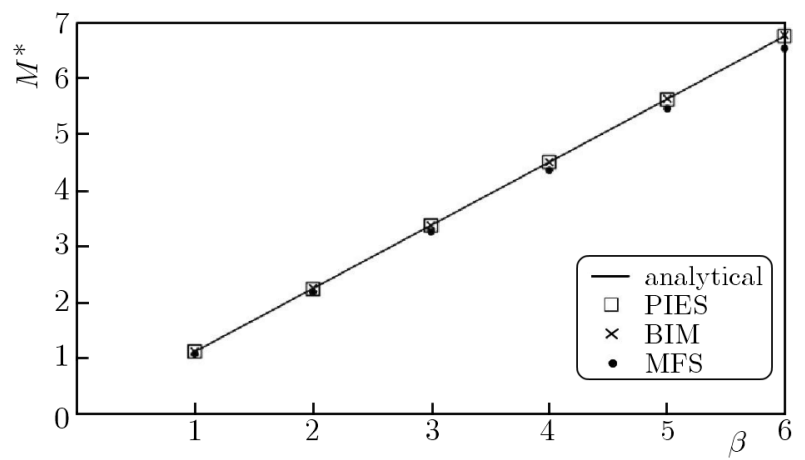

Fig. 8. Comparison of $M^{*}$ for various values of $\beta$

As can be seen in Fig. 8, the values obtained using BIM and PIES are the same as the analytical solutions. The only slight divergence can be observed in the case of the MFS method for $\beta$ greater than 3 . 
Since the approximation strategy has been accurate in the case of the elastic problem, it has been decided to examine its possibilities considering the plastic problem. The solution of such problems requires calculating of the integrals present in (3.1), (3.4) with integrands which require derivatives of plastic strains (2.5) at many points of the domain. Furthermore, the problem is resolved in an iterative process, which involves repeated calculation of the integrals included in the derivative of expression (3.4). Therefore, it has been decided to apply the approximation strategy, which can be done in two ways:

- make the approximation of expressions $\varepsilon_{13}, \varepsilon_{23}$ (2.3) on the basis of the function $u$, using them calculate plastic strains $\varepsilon_{13}^{p}, \varepsilon_{23}^{p}(2.6)$ and then approximate the derivatives from (2.5) - two interpolation polynomials are generated; differentiation is made to obtain the first-order derivatives only,

- make the approximation of expressions $\varepsilon_{13}, \varepsilon_{23}$ (2.3) and derivatives from (2.5) on the basis of the function $u$, expressions (2.7)-(2.10) and similarly generated for the derivative $\varepsilon_{23}^{p}$ with respect to $x_{2}$. One interpolation polynomial is generated; differentiation is made to obtain the derivatives of the first and second order.

In the paper, the second approach is chosen and applied (it is also presented in Fig. 3) because of its efficiency. It is characterized by the fact that at each step of the iterative process, the Lagrange polynomial is obtained only once on the basis of the function $u$ obtained at the interpolation points. Then, only the derivatives of the polynomial with respect to the proper variable and of the proper order are generated.

In order to perform tests, the dimensionless angle of twist per unit length $\beta$ has been then increased in unit steps from 1 to 6 for different values of the strain hardening parameter $m$. Initially, the dimensionless maximum shear stresses $\tau_{\max }^{*}$ for $m=0.2$ have been obtained. For this purpose, we used PIES and the strategy for the approximation with 196 interpolation nodes $(P=R=14)$ placed at roots of the 14th Chebyshev polynomial of the second kind. The obtained results have been compared with solutions from other numerical methods: BIM (Mendelson, 1975) and FDM (Mendelson, 1968) as shown in Table 4.

Table 4. Comparison of $\tau_{\max }^{*}$ obtained by PIES with other numerical methods for $m=0.2$

\begin{tabular}{|c|c|c|c|}
\hline$\beta$ & PIES & BIM & FDM \\
\hline \hline 2 & 0.87558 & 0.879 & 0.881 \\
\hline 3 & 1.01375 & 1.020 & 1.022 \\
\hline 4 & 1.14824 & 1.150 & 1.156 \\
\hline 5 & 1.28215 & 1.280 & 1.290 \\
\hline 6 & 1.4174 & 1.400 & 1.426 \\
\hline
\end{tabular}

As shown in Table 4, the results obtained by PIES using the approximation strategy are very accurate comparing with other numerical methods.

The next examined parameter has been the dimensionless moment $M^{*}$ for $m=0.0,0.1$. This time we compare results with two other numerical methods: BIM (Mendelson, 1975) and MFS (Kołodziej and Gorzelańczyk, 2012). The parameters of PIES and the approximation strategy are taken from the tests presented above. The results are shown in Fig. 9.

Again, the results obtained using PIES are very similar to those obtained by Mendelson (1975) and Kolodziej and Gorzelańczyk (2012). It should be noted that in the proposed approach, the discretization has not been applied. The whole bar cross-section has been modelled by one surface. Therefore, it has not been necessary to calculate and sum the integrals over cells, the global integration over the entire area with a larger number of weights has been used instead. The necessity of calculation of integrals from the differentiated integral identity for displacements has 


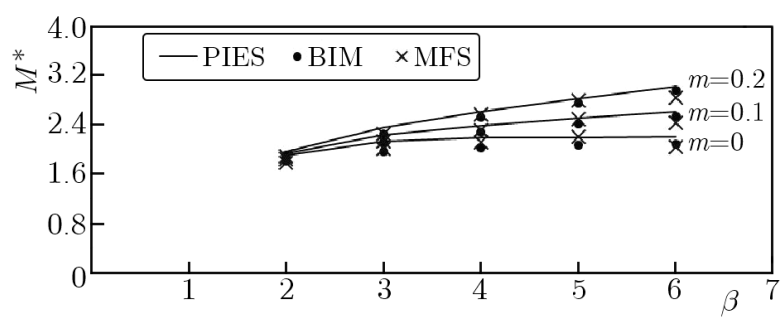

Fig. 9. Comparison of $M^{*}$ for various variants of $\beta$ and $m$

been eliminated and replaced by the numerical approximation of the derivatives of the solution, which is an elementary procedure.

The final stage of the comparative analysis is to examine the effectiveness of the proposed method measured by the number of required iterations. Again, the data are taken from the literature (Mendelson, 1975), therefore, we can compare only two cases:

a) $m=0, \beta=5$ with $\delta=0.0001$,

b) $m=0, \beta=6$ with $\delta=0.0001$ and $\delta=0.00001$.

Table 5 presents the number of iterations required to obtain the solution in case a) with the given level of the accuracy, taking into account three methods: PIES, BIM (Mendelson, 1975) and FDM (Mendelson, 1975). Table 6 contains the number of iterations required for obtaining the solution in case b) taking into account two different levels of the accuracy and two methods: PIES and BIM (Mendelson, 1975). No results are available for the MFS method.

Table 5. The number of iterations in case a)

\begin{tabular}{|c|c|c|}
\hline FDM & BIM & PIES \\
\hline \hline 203 & 33 & 21 \\
\hline
\end{tabular}

Table 6. The number of iterations in the case b)

\begin{tabular}{|c|c|c|}
\hline & BIM & PIES \\
\hline \hline$\delta=0.0001$ & 39 & 25 \\
\hline$\delta=0.00001$ & 53 & 32 \\
\hline
\end{tabular}

As can be noticed in Table 5 and 6, in the considered cases the proposed method requires fewer iterations than the rest of the studied methods. For completeness, we should also examine the execution time. However, it is not possible for the considered problem and included in the manuscript results, because the cited literature does not provide such data. Such tests will be possible to do, if for solving the considered problem we use professional software based on known numerical methods. We will include it in our further research plans.

\section{Conclusions}

The efficient approximation strategy for the derivatives of solutions has been tested on an example of nonlinear torsion of the bar using the PIES method. The application of PIES eliminates the necessity of dividing the considered domain into cells, as it is done in BEM in order to calculate the integrals over the domain. The application of surfaces to define shapes gives the possibility for implementing the global integration with a large number of weights in the Gauss-Legendre quadrature. Moreover, the proposed in the paper approximation strategy enables us 
to avoid repeated calculation of the integrals (often singular) and gives the possibility for effective determination of the derivatives of any order with respect to various variables at any point of the domain and the boundary. It should be emphasized that the obtained results are highly accurate, as shown in comparison to analytical solutions and these obtained by other numerical methods.

We realize that the solved in the paper problem is quite elementary, but we had to test the concept and the mathematical apparatus based on the PIES method. At the next research stage, the gained knowledge makes it possible to apply the strategy and its advantages to more complex nonlinear mechanical problems. In such problems, we will eliminate the necessity of calculating strongly singular integrals, which are present in the integral identity for stresses.

\section{References}

1. Aliabadi M.H., 2002, The Boundary Element Method, Volume 2, Applications in Solid and Structures, John Wiley \& Sons, Ltd., Chichester

2. Ameen M., 2005, Computational Elasticity, Alpha Science International Ltd., Harrow

3. BoŁtuć A., Zieniuk E., 2011, PIES in problems of 2D elasticity with body forces on polygonal domains, Journal of Theoretical and Applied Mechanics, 49, 2, 369-384

4. BoŁtuć A., Zieniuk E., 2013, The effective determination of stress by PIES method using the generalized strategy of derivative approximation (in Polish), Modelowanie Inzynierskie, 16, 47, 41-47

5. Foley J., van Dam A., Feiner S., Hughes J., Phillips R., 1994, Introduction to Computer Graphics, Addison-Wesley

6. Ko€odziej J.A., Gorzelańczyk P., 2012, Application of method of fundamental solutions for elasto-plastic torsion of prismatic rods, Engineering Analysis with Boundary Element, 36, 81-86

7. LiU G.R., 2002, Meshfree Methods - Moving Beyond the Finite Element Method, CRC Press, Boca Raton

8. Mamedov A., 1995, An inverse problem related to the determination of elastoplastic properties of a cylindrical bar, International Journal of Non-Linear Mechanics, 30, 23-32

9. Mendelson A., 1968, Elastic-Plastic Torsion Problems for Strain-Hardening Materials, NASA TN D-4391

10. Mendelson A., 1975, Solution of Elastoplastic Torsion Problem by Boundary Integral Method, NASA TN D-7872

11. Sapountzakis E.J., Tsipiras V.J., 2009, Nonlinear inelastic uniform torsion of composite bars by BEM, Computers and Structures, 87, 3/4, 151-166

12. Sokolnikoff I.S., 1956, Mathematical Theory of Elasticity, McGraw-Hill Book Co. Inc.

13. Sokolowski W. W., 1957, Theory of Plasticity (in polish), PWN, Warszawa

14. Yamada Y., NAKAGiRI S., TAKatsuka K., 1972, Elastic-plastic analysis of Saint-Venant torsion problem by a hybrid stress model, International Journal for Numerical Methods in Engineering, 5 , 2, 193-207

15. ZIENIUK E., 2001, Potential problems with polygonal boundaries by a BEM with parametric linear functions, Engineering Analysis with Boundary Element, 25, 185-190

16. Zieniuk E., 2013, Computational Method PIES for Solving Boundary Value Problems, PWN, Warsaw

17. Zieniuk E., BoŁtuć A., 2006a, Bézier curves in the modeling of boundary geometry for 2D boundary problems defined by Helmholtz equation, Journal of Computational Acoustics, 14, 3, $353-367$ 
18. Zieniuk E., BoŁtuć A., 2006b, Non-element method of solving 2D boundary problems defined on polygonal domains modeled by Navier equation, International Journal of Solids and Structures, 43, 7939-7958

19. Zienkiewicz O., 1977, The Finite Element Methods, McGraw-Hill, London

Manuscript received April 16, 2014; accepted for print August 19, 2014 